\title{
Cancer and Bugs: How much do we know about the Relation between Vaginal Microbiome and Cervical Neoplasia?
}

\author{
Fernandez-Aristi $\mathrm{AR}^{1,2 *}$ and Taco-Masias $\mathbf{A A}^{1,2}$ \\ ${ }^{1}$ School of medicine, Universidad Peruana de Ciencias Aplicadas, Peru \\ ${ }^{2}$ Instituto de Investigacion de Enfermedades Infecciosas (IIEI), Peru
}

Submission: April 16, 2018; Published: July 11, 2018

"Corresponding author: School of medicine, Universidad Peruana de Ciencias Aplicadas, Instituto de Investigacion de Enfermedades Infecciosas (IIEI), Peru, Email: pcmerfer@gmail.com

\begin{abstract}
Cervical cancer is one of the most prevalent gynecologic cancers worldwide and has demonstrated a pathophysiology that involves various factors, but the infection of the Human Papillomavirus (HPV) remains the key stone in the pathogenesis and progression of this neoplasia. Although the colonization by the HPV is mainly driven by sexual transmission, this viral etiology needs to colonize the upper genital tract in order to perpetuate the infection. The Vaginal Microbiome, mainly composed by Lactobacillus Genus, has shown for many years since their discovery protective functions against various microbes which extends from local production of lactic acid, barrier effect, functions as an innate immune response, among others. Recent studies had linked dysbiosis of the Vaginal Microbiome, represented as fewer Lactobacillus strains, to increased risk of colonization of pathologic entities, which the HPV stands as the main etiology. The pro inflammatory state driven by this dysbiosis and the pathologic colonization of HPV, therefore; increase the overall risk of cervical neoplasia. Although this cascade is present in the dysbiotic state, some Lactobacillus strains promotes the HPV colonization and progression to cervical neoplasia.In this mini review we present the current knowledge of how the dysbiosis of the Vaginal Microbiome increased the risk of colonization of HPV and how some Lactobacillus strains and other bacteria in increase the overall risk of cervical cancer.
\end{abstract}

Keywords: Vaginal microbiome; Lactobacillus; Dysbiosis; Human papillomavirus; Inflammation; Cervical neoplasia

\section{Introduction}

One of the current paradigms in microbiology that transcends to all human related science fields are a group of bacteria that colonizes mucosal surfaces, such as the female genital tract (FGT), among the body called the microbiome [1]. A wide variety of bacterial groups that accounts this community has specific and beneficial functions such as a barrier exerting mucosal protection, maturation of immune function and production of soluble factors against microbial infection [2]. In order to achieve this functions, the microbiota; in this case, the women genital microbiota, the balance of diversity and richness are crucial. Any alteration in its homeostasis causes an inflammatory state that promotes microorganism infection [3] and creates a positive loop, where the pathologic colonization creates an increased inflammatory response; further disrupting the vaginal microbiome (VM) [4]. This state is called dysbiosis and it has been linked to pathologies such as Bacterial Vaginosis (VB) [5], increased risk of HIV infection [6] and two gynecologic cancer: Cervical and Endometrial Neoplasias $[7,8]$ in where the disbalanced state of the microbiota promotes etiological factor colonization and progression through the inflammatory state exerted by immune responses in this pathologies. On this matter, the main objective of this review is to compilate the current knowledge regarding the key role of the VM in the pathogenesis and the progression of tumorigenesis in cervical cancer.

\section{Lactobacillus Characterization and Immune} Responses in the Healthy Vaginal Microbiome

Nearly 130 strains of Lactobacillus spp. are detected across the human body, but only 20 of them reside in the FGT [9] and most of the members in this genus are englobed in 4 particular strains: Lactobacillus Inners, Lactobacillus Crispatus, Lactobacillus Jenseii and Lactobacillus Gasseri [10]. It is not completely unraveled why only these 4 strains are able to survive in the vaginal mucosa. To elucidate this issue, a previous study revealed that the genetic pool of those species are smaller and had fewer $\mathrm{G}+\mathrm{C}$ in its genotype compared with other Lactobacillus. In addition, 
the genetic traits of higher mucous binding proteins and lack of amino acids-generating enzymes are the most prevalent traits in these strains [11], meaning that those species can't survive for themselves and need a larger enzyme machinery to metabolize large polymers such as glycogen. A previous review by Nunn $\mathrm{K}$, et al. exposed this topic stating that the Lactobacillus can only absorb this molecule if the vaginal mucous secretions presented $\alpha$-Amylase to break down the glycogen secreted by the caliciform cells [12].

As stated before, these four Lactobacilli have characteristic genetic traits that mostly explain its preference for the mucosa present in the FGT. In exchange, these bacteria exert different protective functions for the host, which functions as innate immune system. Aside from the barrier-like protection in the mucosal surface, these functions are the production of lactic acid which acidifies the FGT maintaining the $\mathrm{pH}$ levels between $3.74 \pm 0.29$ [13], secretion of soluble factors such as bacteriocins and Hydrogen Peroxide [14] thus preventing the colonization of pathogens such as Neisseria Gonorrhoeae, Staphylococcus Aureus, Escherichia Coli, among others $[3,15]$. Other protective function are the interactions of the Lactobacilli with epithelial and mucous membrane receptors, inhibiting the adherence of pathogenic bacteria and viruses and englobing those pathogens reducing their ability to proliferate [16].

Vaginal Microbiome in Genital Infections: Innate and Adaptive Response

The execution of the defense mechanisms stated above cannot be performed if the predominant Genus in the VM is not the Lactobacillus. This state, evaluated with a Nugent score $>3$ which indicates decreased concentration of Lactobacillus strains [17], is driven by the alteration in the vaginal environment undergone by several factors such as a decreased concentration of hormones during menopause and menstruation, age, pregnancy and vaginal infections $[10,12,14]$. After the disbalanced state settles in the VM, colonization of pathogenic Microbiome such as Gardnerella, Mobilluncus, Prevotella, Corynebacterium, Staphylococcus, among others $[9,10]$ that predisposes a various array of Sexual transmitted infections (STI's), in which the Human Papillomavirus (HPV) is included [18]. The innate conformed mainly by Neutrophils, Macrophages and Dendritic cells, localized in the upper FGT [19], along with Pattern recognition receptors (PPR) located in the mucosal epithelia [19,20]; exert the first inflammatory response against the colonization of the HPV. This first response against is followed by the interplay between the dendritic cells and Th2 cells along with T memory cells that initiate IgG secretion, the main soluble factor produced in the vaginal fluid [21]. Both responses helps perpetrate the inflammatory state in vaginal dysbiosis and helps promote the epithelial dysplasia by the HPV [22].

Although this pro inflammatory state is sufficient to promote the colonization and progression of the HPV, other factor play an important role in the variability of the risk of Cervical Neoplasia.
A previous study [23] performed in 70 healthy women with both HPV-negative and HPV-positve showed that Lactobacillus Gasseriwas related to increased HPV detection. In contrast, other studies [24,25] showed that Lactobacilli supernants were related with increased clearance of HPV and inhibitory mechanisms through HPV proteins, E6 and E7. More studies need to be performed to unravel the real interplay between the Lactobacillus Gasseri and the HPV infection.

\section{Colonization by Human Papiloma Virus (Hpv) and Cervical Cancer}

The VM is essential to maintain the balance of bacteria in the female genital tract, any disruption of these bacterial communities leads to greater risk of miscarriage, recurrent abortion, HPV infection, and sexually transmitted infections such HIV, CMV, Chlamydia Trachomatis, among other etiologies. Currently, the association between the VM and the HPV infection is poorly understood. On this

matter, many studies have tried to elucidate the role of VM in the process of this precancerous infection.

Regarding HPV infection, a study done by Lee JE et al. found that HPV-positive women had significantly higher microbial diversity with a lower proportion of Lactobacillus spp. than HPV-negative women. In addition, they found that Fusobacteria is highly associated with HPV infection. Furthermore, they conclude that there are many factors which can influence the VM such as host physiology, genetic factors, and menopause status. Finally, they also identified Sneathia spp.as a microbiological marker of HPV infection [26]. Another study done in Nigerian women evaluated the composition of VM and prevalent high risk of HPV infection. The authors found a moderate association between prevalence of high risk of HPV and a low relative abundance of Lactobacillus sp. Additionally, they found that there was an increased proportion of women with composed of a diverse array of facultative and strictly anaerobic bacteria such as Atopobium and Gardnerella vaginalis, in those who had an HPV infection compared to those without any HPV infection [27].

Likewise, a meta-analysis done by Guillet et al. has reported an association between bacterial vaginosis and HPV infection. The authors explain that loss of Lactobacillus facilitates survival of other sexually transmitted agents such as HPV. This factor along with others such as decreased $\mathrm{pH}$, increased mucindegrading enzymes play an important role in the degradation of the gel layer coating the cervical epithelium, causing cervical micro-abrasions and alterations of the cervical epithelial cells. All of these increase the susceptibility to cervical HPV infection by facilitation the invasion and adherence of HPV genes into the genome of cells of the transformation zone [28].

These results are similar to that obtained in the previously mentioned study. All of this results are according to the literature, especially those bacteria which are decrease such as Lactobacillus sp., since pathophysiologically, its decreasing leads 
to a vaginal dysbiosis, predisposing to get any infection such as HPV. It is important to understand that despite the determinants of the VM composition are not well known. The variations of some factors such as ethnic groups may reflect a role for genetic factors without rule out the non-genetic factors such as sociocultural and health practices [29].

Regarding cervical cancer, according to the literature, VM is important to maintain the stabilization in the female genital tract, but when this VM can change if the body is under an inflammatory process such as cervical cancer. On this matter, it is known that its main predisposing factor is the HPV infection (especially type 16 and 18). A review done by Chase et al. concluded that Lactobacillus Gasseri and Gardnerella vaginalis were found in significantly high frequencies in HPV positive patients in contrast to those with HPV-negative infection [30].

Many studies have been done in high-risk populations in which have found that there are many mechanisms implicated in cervical cancer. One of these is the DNA oxidative damage in which Piyathilake et al. state that this mediates the effect of the microbiome on the natural history of HPV infection and the severity of the high-risk cervical intraepithelial neoplasia (CIN 2). Despite they did not show a strong association between microbiome diversity and CIN severity, they provide suggestive evidence that the VM characterized by the predominance of Lactobacillus is associated with CIN 2 in women infected with high-risk HPV [31]. However, another study done by Mitra et al. found that advancing CIN disease severity is associated with increasing vaginal microbiota diversity and may be involved in regulating viral persistence and disease progression [32].

On this matter, both studies agree that unhealthy vaginal flora is often characterized by high diversity bacterial populations with an increased anaerobic bacteria and lower levels of lactobacillus. All of these are related to pro-inflammatory cytokine concentrations, especially IL-8 and TNF-alpha. Moreover, other bacterial populations play a key role in the development of cervical cancer. Some of these are Proteobacteria and Fusobacteria which metabolize the B12 synthesis of cobalamin, and Bacteroidetes which metabolizes the folate biosynthesis. All of these micronutrients are important because VM may influence the natural history of HPV infection and CIN by altering the status of these in the cervical environment [31].

Despite all previously mentioned, the microbial communities in the cervical mucosa have not been well studied, but we can say that it is plausible that the VM modify the risk of developing cervical cancer along other risk factors such as socio-economic, health and genetic factors [30,31].

\section{Conclusion}

Foundational studies are increasing largely, as well as emerging evidence is increasing to suggest that the vaginal microbiota plays a substantial role in the persistence of the virus and the presence of subsequent cervical pre-invasive disease.
Future studies should focus on elucidating the pathophysiology and the main pathways in which the vaginal microbiota are present and the way it can change the course of many inflammatory processes such as cervical cancer. These studies should aim to elucidate the complete understanding of the relationship of vaginal microbiota and cervical cancer, especially in risk populations.

\section{References}

1. Llyod-Price J, Abu-Ali G, Huttenhower (2016) The healthy human microbiome. Genome med 8: 51.

2. Llyod-Price J, Mahurkar A, Rahnavard G, Crabtree J, Orvis J, et al. (2017) Strains, functions, and dynamics in the expanded Human Microbiome Project. Nature 550(7674): 61-66.

3. Anahtar M, Byrne E, Doherty K, Bowman B, Yamamoto H, et al. (2015) Cervicovaginal bacteria are a major modulator of host inflammatory responses in the female genital tract. Immunity 42(5): 965-976.

4. Doerflinger SY, Throop AL, Herbst-Kralovetz MM (2014) Bacteria in the vaginal microbiome alter the innate immune response and barrier properties of the human vaginal epithelia in a species-specific manner. J Infect Dis 209(12): 1989-1999.

5. Vitali B, Cruciani F, Picone G, Parolin C, Donders G, et al. (2015) Vaginal microbiome and metabolome highlight specific signatures of bacterial vaginosis. Eur J Clin Microbiol Infect Dis 34(12): 2367-2376.

6. Petrova MI, Broek VDM, Balzarini J, Vanderleyden J, Lebeer S, et al. (2013) Vaginal microbiota and its role in HIV transmission and infection. FEMS Microbiol Rev 37(5): 762-792.

7. Kyrgiou M, Mitra A, Moscicki AB (2017) Does the vaginal microbiota play a role in the development of cervical cancer? Transl Res 179: 168182.

8. Walther-António MR, Chen J, Multinu F, Hokenstad A, Distad TJ, et al. (2016) Potential contribution of the uterine microbiome in the development of endometrial cancer. Genome Med 8(1): 122.

9. Fettweis J, Brooks JP, Serrano M, Sheth N, Girerd P, et al. (2014) Differences in vaginal microbiome in African American women versus women of European ancestry. Microbiology 160(pt 10): 2272-2282.

10. Huang B, Fettweis J, Brooks JP, Jefferson K, Buck G, et al. (2014) The Changing Landscape of the Vaginal Microbiome. Clin Lab Med 34(4): 747-761.

11. Mendes-Suarez H, Suziki H, Hickey R, Forney L (2014) Comparative Functional Genomics of Lactobacillus spp. Reveals Possible Mechanisms for Specialization of Vaginal Lactobacilli to Their Environment. J Bacteriol 196(7): 1458-1470.

12. Nunn K, Forney L (2016) Unravelling the dynamics of the human vaginal microbiome. Yale J Biol Med 89(3): 331-337.

13. O'Hanlon D, Moench T, Cone R (2013) Vaginal pH and Microbicidal Lactic Acid When Lactobacilli Dominate the Microbiota. PLoS ONE 8(11): e80074.

14. Green K, Zarek S, Catherino W (2015) Gynecologic health and disease in relation to the microbiome of the female reproductive tract. Fertil Steril 104(6): 1351-1357.

15. Ghartey J, Smith B, Chen Z, Buckley N, Lo Y, et al. (2013) Lactobacillus crispatus Dominant Vaginal Microbiome Is Associated with Inhibitory Activity of Female Genital Tract Secretions against Escherichia coli. PLOS ONE 9(5): e96659.

16. Borges S, Silva J, Teixeira P (2013) The role of lactobacilli and probiotics in maintaining vaginal health. Arch Gynecol Obstet 289(3): 479-489. 
17. Amegashie C, Gilbert N, Peipert J, Allsworth J, Lewis W, et al. (2017) Relationship between nugent score and vaginal epithelial exfoliation. PLoS ONE 12(5): e0177797.

18. Nardis C, Mosca L, Mastromarino P (2013) Vaginal microbiota and vira sexually transmitted diseases. Ann Ig 25(5): 443-456.

19. Brotman R, Ravel J, Bavoil, P, Gravitt P, Ghanem K, et al. (2014) Microbiome, Sex Hormones, and Immune Responses in the Reproductive Tract: Challenges for Vaccine Development Against Sexually Transmitted Infections. Vaccine 32(14): 1543-1552.

20. Wira CR, Ghosh M, Smith JM, Shen L, Connor RI, et al. (2011) Epithelial cell secretions from the human female reproductive tract inhibit sexually transmitted pathogens and Candida albicans but not Lactobacillus. Mucosal immunology 4(3): 335-342.

21. Mestecky J, Raska M, Novak J, Alexander RC, Moldoveanu Z, et al. (2010) Antibody-mediated protection and the mucosal immune system of the genital tract: relevance to vaccine design. J Reprod Immunol 85(1): 8185 .

22. Petry KU (2014) HPV and cervical cancer. Scand J Clin Lab Invest Suppl 244: 59-62.

23. Gao W, Weng J, Gao Y, Chen X (2013) Comparison of the vagina microbiota diversity of women with and without human papillomavirus infection: a cross-sectional study. BMC Infect Dis 13: 271

24. Brotman RM, Shardell MD, Gajer P, Tracy JK, Zenilman JM, et al. (2014) Interplay between the temporal dynamics of the vaginal microbiota and human papillomavirus detection. J Infect Dis 210(11): 1723-1733.
25. Wang KD, Xu DJ, Wang BY, Yan DH, Lv Z, et al. (2018) Inhibitory Effect of Vaginal Lactobacillus Supernatants on Cervical Cancer Cells. Probiotics Antimicrob Proteins 10(2): 236-242.

26. Lee JE, Lee S, Lee H, Yun-Mi S, Kayoung L, et al. (2013) Association of the vaginal microbiota with human papilloma virus in a Korean twin cohort. PLoS One 8(5): e63514.

27. Dareng EO, Ma B, Famooto AO, Adebamowo SN, Offiong RA, et al. (2016) Prevalent hugh-risk HPV infection and vaginal microbiota in Nigerian women. Epidemiol Infect 144(1): 123-137.

28. Gillet E, Meys JF, Verstraelen H, Bosire C, Sutter DP, et al. (2011) Bacterial vaginosis is associated with uterine cervical human papillomavirus infection: a meta-analysis. BMC Infect Dis 11: 10 .

29. Ravel J, Gajer P, Abdo Z, Schneider GM, Koenig SS, et al. (2011) Vaginal microbiome of reproductive-age women. Proc Natl Acad Sci USA 108 (Suppl 1): 4680-4687.

30. Chase D, Goulder A, Zenhausern F, Monk B, Herbst KM, et al. (2015) The vaginal and gastrointestinal microbiomes in gynecologic cancers: A review of applications in etiology, symptoms and treatment. Gynecol Onco 138(1): 190-200.

31. Piyathilake CJ, Ollberding NJ, Kumar R, Macaluso M, Alvarez RD, et al. (2016) Cervical Microbiota Associated with Higher Grade Cervical Intraepithelial Neoplasia in Women infected with High-Risk Human Papillomaviruses. Cancer Prev Res (phila) 9(5): 357-366.

32. Mitra A, MacIntyre DA, Lee YS, Smith A, Marchesi JR, et al. (2015) Cervical intraepithelial neoplasia disease progression is associated with increased vaginal microbiome diversity. Sci Rep 5: 16865.

\section{Your next submission with Juniper Publishers will reach you the below assets}

- Quality Editorial service

- Swift Peer Review

- Reprints availability

- E-prints Service

- Manuscript Podcast for convenient understanding

- Global attainment for your research

- Manuscript accessibility in different formats

( Pdf, E-pub, Full Text, Audio)

- Unceasing customer service

Track the below URL for one-step submission https://juniperpublishers.com/online-submission.php 